\section{Level of Anticitrullinated Peptide/Protein Antibody Is Not Associated with Lung Diseases in Rheumatoid Arthritis}

\section{To the Editor:}

The correlation between anti-cyclic citrullinated peptide (CCP) and lung diseases in patients with rheumatoid arthritis (RA) has been the subject of attention since anti-CCP was introduced. Recently, Aubart and colleagues reported that high anti-CCP2 levels were independent risk factors for and associated with lung diseases in patients with $\mathrm{RA}^{1}$. However, other authors reported no association between anti-CCP and rheumatic lung disease ${ }^{2,3}$.

We retrospectively investigated lung diseases in anti-CCP-positive RA patients. However, we found no differences of anti-CCP level between RA patients with or without lung disease. To explain the difference of our results, we suspected a methodologic limitation of the enzyme linked immunosorbent assay (ELISA) used for the anti-CCP test, because we have previously reported that failure of the correlation between serum ferritin level and presence of anti-CCP in RA patients was corrected by antiperinuclear factor $(\mathrm{APF})^{4}$. Therefore we studied the association between RA lung disease and APF, and evaluated RA patients that had both of these tests.

From January 2008 to January 2011, 411 patients with RA that were positive for either anti-CCP or APF were investigated by reviewing medical records including simple chest radiographs and chest high-resolution computed tomography (HRCT) scans. Lung diseases were classified, i.e., rheumatic lung diseases, including interstitial pulmonary fibrosis, bronchiectasis, and rheumatoid nodules, and other lung diseases. Anti-CCP was performed by second-generation ELISA (Axis-Shield, Scotland or Immco Diagnostics, Buffalo, NY, USA) and levels $>25 \mathrm{U} / \mathrm{ml}$ were considered positive. APF test was by the indirect immunofluorescence method using a commercial kit (IT-APF ${ }^{\mathrm{TM}}$ ImmunoThink Co., Seoul, Korea) and interpreted from level 1 to 4 according to fluorescence intensity.

Rheumatic lung disease in our RA patients was $7.6 \%$, lower than the $23.4 \%$ of the authors' data, and $16.1 \%$ even if accounting for all the lung diseases (Table 1). In RA patients positive for 2 ACPA tests, the anti-CCP level of those without lung disease $(723 \mathrm{U} / \mathrm{ml})$ was higher compared to patients with rheumatic lung disease $(538 \mathrm{U} / \mathrm{ml})$ or patients with other lung disease $(681 \mathrm{U} / \mathrm{ml})$, and a similar phenomenon was observed in RA patients positive only for anti-CCP (Table 2). As for the fluorescence intensity findings for APF in RA patients positive for 2 ACPA tests, the median level of patients with rheumatic lung disease was slightly higher than that for patients without rheumatic lung disease, but the difference was not statistically significant. And there were no statistically significant differences in treatment features including use of anti-tumor necrosis factor- $\alpha$ inhibitors among the 3 subgroups of patients with RA.

The differences between our results and those of Aubart, et al ${ }^{1}$ would represent early-stage disease diagnosed only by HRCT, because our data included not only HRCT but also simple chest radiograph findings compared with the authors' data that all patients were examined with HRCT. However, the authors' data did not show the specific number of lung diseases detected only by HRCT. Although it is difficult to estimate how many patients are relevant to this condition, we consider many patients were not appropriate. In addition, our data has the limitation that it did not objectively compare disease durations among 3 subgroups of RA patients, because in some patients it was difficult to determine the exact time of the start of symptoms. However, the 3 groups of patients showed a similar configuration, so we assume there are no significant differences. Finally, the possibility of ethnic differences is presumed by those showing differences in prevalence of lung disease, and the underestimation of rheumatoid nodules is likely because South Korea is an endemic area of tuberculosis.

In summary, we found that levels of ACPA by the 2 methods did not reflect the presence of lung disease in patients with RA. Therefore, we believe that further investigation is required to determine whether the level of anti-CCP can be regarded as a good indicator of the lung involvement in patients with RA.

LA-HE JEARN, MD, Associate Professor; THINK-YOU KIM, MD, Professor, Department of Early Arthritis/Laboratory Medicine, The Hospital for Rheumatic Diseases, Hanyang University Medical Center, 17 Haengdang-Dong, Seongdong-Gu, Seoul, 133-792, Republic of Korea. Address correspondence to Dr. T-Y. Kim; E-mail: tykim@hanyang.ac.kr

Table 1. Distribution of lung disease in ACPA-positive patients with RA.

\begin{tabular}{lccc}
\hline \multirow{2}{*}{ Patient Group } & & \multicolumn{2}{c}{ RA with Lung Disease } \\
Other Lung Disease**, $\mathrm{n}$ & Total \\
\hline APF+, anti-CCP+ & RA without Lung Disease, $\mathrm{n}$ & 16 & 15 \\
APF+, anti-CCP- & 199 & 1 & 7 \\
APF-, anti-CCP+ & 12 & 14 & 230 \\
Total & $345(83.9 \%)$ & $31(7.6 \%)$ & 13 \\
\hline
\end{tabular}

* Rheumatic lung disease includes interstitial pulmonary fibrosis, bronchiectasis, and rheumatoid nodules. ** Includes pulmonary involvement such as tuberculosis, atelectasis, pneumonia, focal inflammation, excepting rheumatic lung diseases. ACPA: anticitrullinated peptide/protein antibody; RA: rheumatoid arthritis; APF: antiperinuclear factor; CCP: cyclic citrullinated peptide.

Table 2. Median values of ACPA level according to lung disease in ACPA-positive patients with RA

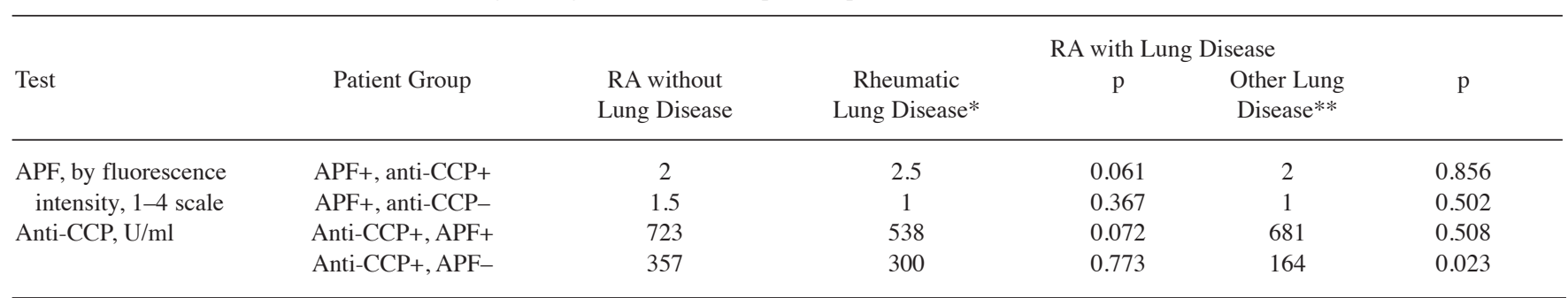

* Rheumatic lung disease includes interstitial pulmonary fibrosis, bronchiectasis, and rheumatoid nodules. ** Includes pulmonary involvement such as tuberculosis, atelectasis, pneumonia, focal inflammation, excepting rheumatic lung diseases. ACPA: anticitrullinated peptide/protein antibody; RA: rheumatoid arthritis; APF: antiperinuclear factor; CCP: cyclic citrullinated peptide. 


\section{REFERENCES}

1. Aubart F, Crestani B, Nicaise-Roland P, Tubach F, Bollet C, Dawidowicz K, et al. High levels of anti-cyclic citrullinated peptide autoantibodies are associated with co-occurrence of pulmonary diseases with rheumatoid arthritis. J Rheumatol 2011;38:979-82.

2. Korkmaz C, Us T, Kasifoglu T, Akgun Y. Anti-cyclic citrullinated peptide $(\mathrm{CCP})$ antibodies in patients with long-standing rheumatoid arthritis and their relationship with extra-articular manifestations. Clin Biochem 2006;39:961-5.

3. Inui N, Enomoto N, Suda T, Kageyama Y, Watanabe H, Chida K. Anti-cyclic citrullinated peptide antibodies in lung diseases associated with rheumatoid arthritis. Clin Biochem 2008;41:1074-7.

4. Kim DA, Kim TY. Variable association of anti-CCP positivity with serum ferritin may be corrected by APF test. Rheumatol Int 2010;30:997-8.

J Rheumatol 2012;39:7; doi:10.3899/jrheum.111478 